\title{
Multiple Source Localization Based on Biased Bearings Using the Intensity Filter - Approach and Experimental Results
}

\author{
Marek Schikora, Marc Oispuu, Wolfgang Koch \\ Fraunhofer FKIE, Dept. Sensor Data and Information Fusion \\ Neuenahrer Straße, 53343 Wachtberg, Germany \\ \{marek.schikora, marc.oispuu,wolfgang.koch\}@fkie.fraunhofer.de
}

\author{
Daniel Cremers \\ TU Munich, Dept. Computer Science \\ Boltzmannstr. 3, 85748 Garching, Germany \\ daniel.cremers@in.tum.de
}

\begin{abstract}
This paper investigates the three-dimensional localization problem for multiple emitters using a realistic airborne array sensor. In order to achieve improved results systematic and statistical direction finding errors are considered in a unified algorithm. The task is solved using a sequential Monte Carlo (SMC) implementation of the intensity filter (iFilter). In this paper, we compare two localization approaches without and with the consideration of systematic bearing errors and verify them with an experimental data set. The comparison of both approaches reveals that the bias consideration offers a superior performance.

Index Terms-Source localization, multiple-target tracking,
\end{abstract} intensity filter, bias estimation

\section{INTRODUCTION}

The localization of multiple sources using passive direction finding (DF) sensors is a fundamental task encountered in various fields like wireless communication, radar, and sonar. Passive DF in contrast to active methods does not transmit known signals to illuminate the scene and process reflected signals, i.e. passive DF methods deliver the signal direction of arrival (DOA) but no range information. Bearings-only localization (BOL) can be realized through multiple DF sensors or a single moving DF sensor. In this work, we consider a single DF sensor mounted beneath an airborne sensor platform (Fig. 1). Aspects of the BOL problem examined in the literature include algorithms, accuracy, and target observability. Two important ways to solve the multiple source BOL problem are batch algorithms and recursive, filter-based methods [1, and refs. cited therein].

In the literature, often idealistic DF sensors are supposed that collect bearings measurements without systematic errors, but in practice, measurements collected by a realistic DF sensor include both statistical as well as systematical errors. Navigation errors in the own position or attitude, an unknown attitude displacement between DF sensor and navigation sensor, or a misspecified sensor model can lead to systematic bearing errors. In [2], the Cramér-Rao bound on the source location estimation error based on biased bearings has been derived. For example in [3], the localization problem has been solved using biased range and bearing measurements obtained by a multi-sensor network. The problem has been solved by a

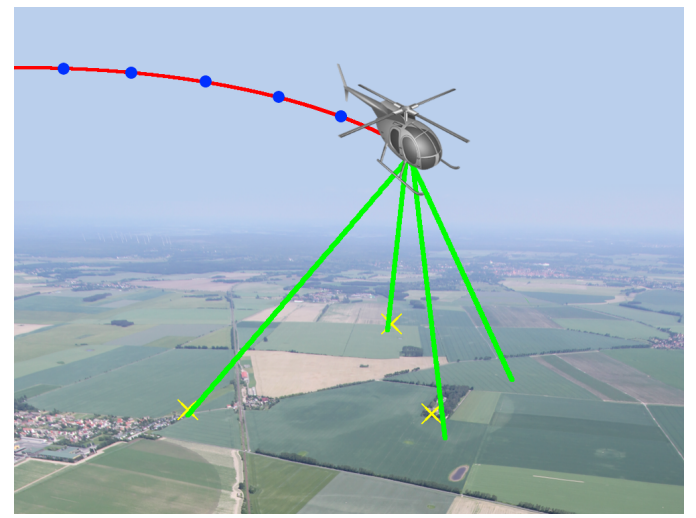

Fig. 1. Considered localization scenario with multiple emitters (yellow crosses) a single moving sensor moving collecting bearing measurements (green lines) at different points in space (blue dots) along the sensor path (red curve)

Kalman filter using an augmented state vector containing the source location and the sensor bias.

In our previous work, we considered bearings measurements obtained by an airborne image sensor or a compact antenna array sensor [4], [5]. In [4], we used an SMC implementation of the intensity filter ${ }^{1}$ (iFilter) to estimate the individual source states. Assuming an ideal DF sensor, the iFilter has been applied to bearing measurements originated by a realistic DF sensor in order to solve the BOL problem. In [7], we considered a realistic array sensor with systematic errors that depend on the signal DOA itself. Furthermore, we presented a batch algorithm that takes the sensor bias into account without explicitly computing the sensor bias.

In this work, we combine both aspects of our previous work to overcome the drawback of the batch algorithm w.r.t. a varying number of emitters and the presence of false targets. Therefore, we briefly review the iFilter and show how to consider systematic sensor errors in this approach. In the

\footnotetext{
${ }^{1}$ The iFilter was derived through Poisson point processes (PPPs) as a generalization of the probability hypothesis density (PHD) filter [6]. PPPs are very useful theoretical models to determine and to track an unknown number of targets.
} 
following, this approach is referred to as bias-iFilter. The proposed method has been verified with real data obtained by a compact array sensor in flight experiments. The results show that the bias-iFilter approach outperforms the iFilter approach.

The paper is organized as follows: In Section II, we formulate the localization problem including the systematic bearing error. In Section III, we outline the considered localization techniques based on the iFilter. In Section IV, we compare the accuracy of both approaches using a real experimental data set. Finally, the conclusions are given in Section V.

\section{Problem Statement}

The following three-dimensional BOL problem is considered (Fig. 1): A single airborne observer equipped with a DF sensor moves along an arbitrary but known trajectory $\mathbf{r}(t)$. The sensor observes $Q$ ground-located sources at the positions $\mathbf{p}_{q}$, $q=1, \ldots, Q$. During the movement the sensor collects multiple bearings at different points in space at time $t_{k}, k=1,2, \ldots$. The relative vector between the sensor at some time $t_{k}$ and some source location $\mathbf{p}$ is given by

$$
\triangle \mathbf{r}_{k}(\mathbf{p})=\mathbf{r}\left(t_{k}\right)-\mathbf{p} .
$$

Commonly, the source-to-sensor bearing measurements of the DF sensor are defined by azimuth $\alpha$ and elevation $\varepsilon$. The earthfixed bearings are nonlinearly related to the source location p. In the absence of noise and interference, the measurement vector satisfies

$$
f_{k}(\mathbf{p})=\left(\begin{array}{l}
\alpha_{k}(\mathbf{p}) \\
\varepsilon_{k}(\mathbf{p})
\end{array}\right)=\left(\begin{array}{c}
\arctan \frac{\triangle x_{k}}{\triangle y_{k}} \\
\arctan \frac{\triangle z_{k}}{\sqrt{\triangle x_{k}^{2}+\triangle y_{k}^{2}}}
\end{array}\right),
$$

where $\triangle x_{k}, \triangle y_{k}$, and $\triangle z_{k}$ denote the components of the relative vector (1). Assuming bearing noise, the bearing measurements that correspond to a source at location $\mathbf{p}$ are given by

$$
\mathbf{z}_{k}=f_{k}(\mathbf{p})+\mathbf{w}_{k},
$$

where the bearing measurements of a realistic DF sensor are considered that are assumed to be normal distributed and biased, i.e. $\mathbf{w}_{k} \sim \mathcal{N}\left(\mathbf{b}_{k}, \mathbf{C}_{k}\right)$. Therein, $\mathbf{b}_{k}=\left(b_{\alpha, k}, b_{\varepsilon, k}\right)^{T}$ denotes the unknown sensor bias and $\mathbf{C}_{k}=\operatorname{diag}\left(\sigma_{\alpha, k}^{2}, \sigma_{\varepsilon, k}^{2}\right)$ the bearing error covariance. In practice, the bearing covariance is unknown and needs to be estimated for localization purposes, e.g. [7, Eq. (10)]. Also the sensor bias depends on the measurement time $t_{k}$. In fact for the considered sensor, the sensor bias is direction dependent and changes slightly during the movement of the sensor. In this work, we consider two alternative state vectors $\mathrm{x} \in \mathcal{S}$, where $\mathcal{S}$ denotes the state space:

$$
\mathbf{x}=\left\{\begin{array}{ll}
\mathbf{p} & , \text { only location } \\
\left(\mathbf{p}^{T}, \mathbf{b}^{T}\right)^{T} & , \text { location and sensor bias }
\end{array} .\right.
$$

Since the sources transmit intermittently and the sensor signal detection may be inaccurate, the sensor collects at time $t_{k}$ a set of $n_{k}$ bearing measurements. Furthermore, the total number of sources is unknown and may vary over long observation periods. The index set of emitting sources at time $t_{k}$ is denoted by the index set $I_{k} \subseteq\{1, \ldots, Q\}$ and the state set as well as the measurement set by

$$
\begin{aligned}
\mathcal{X}_{k} & =\left\{\mathbf{x}_{q} \mid q \in I_{k}\right\}, \\
\mathcal{Z}_{k} & =\left\{\mathbf{z}_{k}\left(\mathbf{p}_{q}\right) \mid q \in I_{k}\right\} \cup \mathcal{Z}_{\phi, k} \\
& =\left\{\mathbf{z}_{1}, \ldots, \mathbf{z}_{n_{k}}\right\},
\end{aligned}
$$

where $\mathcal{Z}_{\phi, k}$ denotes a set of bearing measurements that correspond to clutter targets. Analogous to [4], the multi-target process $\mathcal{X}_{1}, \mathcal{X}_{2}, \ldots, \mathcal{X}_{k}$ can be approximated by a Poisson point process (PPP). With this the measurements $\mathcal{Z}_{1}, \mathcal{Z}_{2}, \ldots, \mathcal{Z}_{k}$ are a realization of a PPP and can be also approximated by a PPP.

The localization problem is stated as follows: Estimate all source locations $\mathbf{p}_{q}, q=1, \ldots, Q$, from a growing number of measurements $\mathcal{Z}_{k}, k=1,2, \ldots$.

\section{LOCALIZATION APPROACH}

The flow graph of our algorithm can be seen in Fig. 2 . The used SMC implementation of the iFilter can be summarized in six steps (blue blocks). The iFilter circumvents the bearing data association inherent in conventional localization approaches and determines also the number of sources. The key idea is to represent the target intensity by particles at each time instant $t_{k}$. Furthermore, the intensity of the clutter space $\phi$ is denoted by $f_{k-1 \mid k-1}(\phi)$. In practical implementations this intensity can be represented by a single number, called the number of $\phi$ hypotheses. Details about the iFilter can be found in [4]. In order to model the filter fully the following probabilities have to be defined:

$$
\begin{aligned}
\psi_{k}(\mathbf{x} \mid \phi) & \text { transition probability for new targets, } \\
\psi_{k}(\phi \mid \phi) & \text { transition probability in } \phi, \\
\psi_{k}(\phi \mid \mathbf{x}) & \text { transition probability for target death, } \\
p_{k}(\mathbf{z} \mid \phi) & \text { probability for measurement from clutter, } \\
p_{k}^{\mathrm{D}}(\mathbf{x}) & \text { detection probability for } \mathbf{x}, \\
p_{k}^{\mathrm{D}}(\phi) & \text { detection probability for } \phi .
\end{aligned}
$$

In this work, two versions of the iFilter without and with the consideration of the sensor bias are compared. The iFilter is initialized with $k=0$. Therefore, the initial state set $\mathcal{X}_{0}$

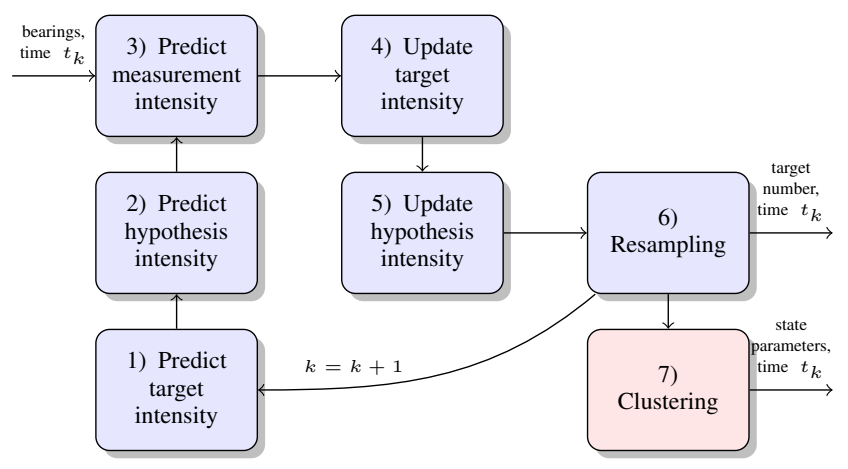

Fig. 2. Basic steps of the proposed localization approach including the iFilter (blue blocks) and the post-processing (red block) 
and the initial values of the aforementioned probabilities must be defined. Finally in a post-processing step, a clustering approach is applied to the iFilter outputs in order to obtain the state parameters and parameter uncertainties. New bearing measurements can be processed within the next iFilter iteration $(k=k+1)$ by continuing with Step 1 .

1) Predict target intensity: At time $t_{k}$, the following set of $N_{k}$ particles is available to represent the target intensity: $\left\{\left(\mathbf{x}_{i}, w_{i}\right)\right\}_{i=1}^{N_{k}}$, where $\mathbf{x}_{i}$ denotes the state vector of the $i$-th particle (4) and $w_{i}$ the corresponding weight. Each particle may represent a possible source state. Since the sources are assumed to be stationary, the particles can be predicted by adding some process noise $\left(\sigma_{\mathbf{p}}, \sigma_{\mathbf{b}}\right)$ to their state while the weights are unchanged. The iFilter models the birth process by itself, so that the particle number has to be increased in order to represent newly born targets correctly. The additional number of particles is given by

$$
N_{k, \text { new }}=N_{k} \psi_{k}(\mathbf{x} \mid \phi) f_{k-1 \mid k-1}(\phi) .
$$

With this, the total number of particles is given by $N_{k \text {,tot }}=$ $N_{k}+N_{k \text {,new }}$. Newly created particles are uniformly distributed over the state space $\mathcal{S}$. The corresponding weight of the $i$-th particle, $i=N_{k}+1, \ldots, N_{k, \text { tot }}$, is given by

$$
w_{i}=\psi_{k}(\mathbf{x} \mid \phi) f_{k-1 \mid k-1}(\phi) N_{k, \text { tot }}^{-1} .
$$

The predicted particle set containing the shifted and newly created particles is defined as $\left\{\left(\mathbf{x}_{i}, w_{i}\right)\right\}_{i=1}^{N_{k, \text { tot }}}$.

2) Predict hypothesis intensity: In order to predict the number of $\phi$ hypotheses, add the number of persistently absent and newly absent targets, so that the predicted number is then:

$$
f_{k \mid k-1}(\phi)=\psi_{k}(\phi \mid \phi) f_{k-1 \mid k-1}(\phi)+\sum_{i=1}^{N_{k, \text { tot }}} \psi_{k}\left(\phi \mid \mathbf{x}_{i}\right) w_{i}
$$

3) Predict measurement intensity: For each measurement $\mathbf{z}_{j}, j=1, \ldots, n_{k}$, compute the partition functions for the source location space as well as the "clutter target" space. The sum of both is the measurement intensity for $\mathbf{z}_{j}$ :

$$
\begin{aligned}
\lambda_{k \mid k-1}\left(\mathbf{z}_{j}\right)=p_{k}\left(\mathbf{z}_{j} \mid \phi\right) & p_{k}^{\mathrm{D}}(\phi) f_{k \mid k-1}(\phi) \\
& +\sum_{i=1}^{N_{k, \text { tot }}} p_{k}\left(\mathbf{z}_{j} \mid \mathbf{x}_{i}\right) p_{k}^{\mathrm{D}}\left(\mathbf{x}_{i}\right) w_{i},
\end{aligned}
$$

with

$$
p_{k}\left(\mathbf{z}_{j} \mid \phi\right)=\frac{f_{k-1 \mid k-1}^{m_{k}}(\phi)}{m_{k} !} \mathrm{e}^{-f_{k-1 \mid k-1}(\phi)}
$$

and where the measurement likelihood function $p_{k}(\mathbf{z} \mid \mathbf{x})$ describes how likely the measurements $\mathbf{z}$ are given the state vector $\mathbf{x}$. In this work, two different state vectors are considered containing only the source location or the additional sensor bias (4). The corresponding likelihood functions are given by

$$
p_{k}(\mathbf{z} \mid \mathbf{x})= \begin{cases}\mathrm{e}^{-\frac{1}{2} M^{2}\left(\mathbf{z}, f_{k}(\mathbf{p}), \mathbf{C}_{k}\right)} & , \mathbf{x}=\mathbf{p} \\ \mathrm{e}^{-\frac{1}{2} M^{2}\left(\mathbf{z}, f_{k}(\mathbf{p})+\mathbf{b}, \mathbf{C}_{k}\right)} & , \mathbf{x}=\left(\mathbf{p}^{T}, \mathbf{b}^{T}\right)^{T},\end{cases}
$$

where $M(\mathbf{z}, \mathbf{m}, \mathbf{C})=\sqrt{(\mathbf{z}-\mathbf{m})^{T} \mathbf{C}^{-1}(\mathbf{z}-\mathbf{m})}$ is the Mahalanobis distance.
4) Update target intensity: Given $n_{k}$ new measurements the update of the state intensity is realized through a correction of the individual particle weights. The particle set with the updated particle intensities is given by $\left\{\left(\mathbf{x}_{i}, h\left(\mathbf{x}_{i}, \mathcal{Z}_{k}\right) w_{i}\right)\right\}_{i=1}^{N_{k} \text { tot }}$ with

$$
h\left(\mathbf{x}, \mathcal{Z}_{k}\right)=1-p_{k}^{\mathrm{D}}(\mathbf{x})+\sum_{j=1}^{n_{k}} \frac{p_{k}\left(\mathbf{z}_{j} \mid \mathbf{x}\right) p_{k}^{\mathrm{D}}(\mathbf{x})}{\lambda_{k \mid k-1}\left(\mathbf{z}_{j}\right)} .
$$

5) Update hypothesis intensity: Since we use the augmented space $\mathcal{S}^{+}$we have to adjust also the number of $\phi$ hypotheses:

$$
f_{k \mid k}(\phi)=h\left(\phi, \mathcal{Z}_{k}\right) f_{k \mid k-1}(\phi),
$$

where $h\left(\phi, \mathcal{Z}_{k}\right)$ is defined analogous to $h\left(\mathbf{x}, \mathcal{Z}_{k}\right)$ using the corresponding probabilities $p_{k}\left(\mathbf{z}_{j} \mid \phi\right)$ and $p_{k}^{\mathrm{D}}(\phi)$.

6) Resampling: Analog to the PHD filter the integral over this intensity is the estimated number of targets $\hat{Q}_{k}$. Since the particle set represents the target intensity of the PPP, the target number can be estimated by the sum over the intensity, i.e.

$$
\hat{Q}_{k}=\sum_{i=1}^{N_{k, \text { tot }}} w_{i}
$$

The number of particles in the state space may and should vary over time in order to represent the current situation better, e.g. more targets need more particles, so that the particle approximation accuracy is still sufficient. The correct number of particles $N_{k+1}$ resampled for the next time step is:

$$
N_{k+1}=\frac{\hat{Q}_{k}}{\hat{Q}_{k}+f_{k \mid k}(\phi)} N_{k, \text { tot }} .
$$

The estimation of $N_{k+1}$ in each time step prevails the particle number from growing against infinity. Given $N_{k+1}$ a standard resampling technique for particle filtering can be used. Rescale the weights by $\hat{Q}_{k}$ to get a new particle set $\left\{\left(\mathbf{x}_{i}, \hat{Q}_{k} / N_{k+1}\right)\right\}_{i=1}^{N_{k+1}}$.

7) Clustering: After each time step $k$ we generate a particle cloud, which represents the PPP over $\mathcal{S}$. To determine the correct source states from this cloud a clustering algorithm (e.g. [8]) can be applied to the particles. Alternatively, the clustering step can be avoided by using the approach in [9].

\section{EXPERIMENTAL RESULTS}

In order to verify our approach, we used both filter strategies on real data gained from flight experiments with a compact antenna array mounted beneath an aircraft. In a pre-processing step, we use the conventional beamformer to calculate the signal bearings. During the movement, the sensor collects bearings that correspond to $Q=3$ stationary sources. The position and attitude of the sensor is measured by the on-board navigation sensor $\left(\sigma_{\text {pos }}=0.5 \mathrm{~m}, \sigma_{\text {att }}=0.1^{\circ}\right)$. Comparing the considered bearing data batch with the ground-truth, the bearings exhibit systematical errors of approximately $b_{\alpha}=5^{\circ}$ and $b_{\varepsilon}=10^{\circ}$. 
In order to solve the BOL problem, we apply the localization approach presented in Section III. The iFilter ${ }^{2}$ is initialized with a set of 15000 particles. The iFilter circumvents the association problem, but in our specific scenario the signal sources can be also identified by their frequency. In the postprocessing step, the $k$-means clustering algorithm has been used.

Regarding just the bearings of the first source, Fig. 3 compares the particle clouds for the iFilter and the biasiFilter for $k=6$ and $k=22$. The localization result of the iFilter can be geometrically interpreted as triangulation, i.e. the bearing lines intersect at the estimated source location. Since the bearing measurements are biased, also the corresponding localization results are biased. For $k=6$, the particles of the bias-iFilter show a circle structure. This behavior can be explained by the observability condition for the BOL problem based on biased bearings. In [2] it is mentioned that a target cannot be localized, if the sensor moves along the line of sight or on a circle through the target location. In our threedimensional case, this considerations lead to an uncertainty along a sphere defined by the sensor path and the source location. In this way, the sensor bias can be compensated with a growing number of measurements.

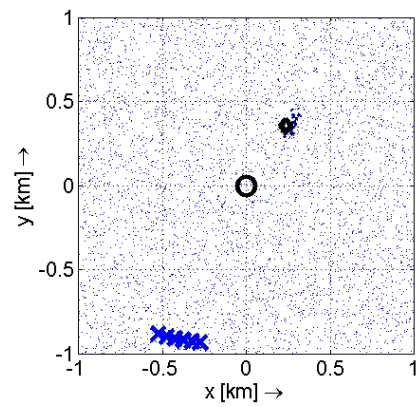

(a) iFilter, $k=6$

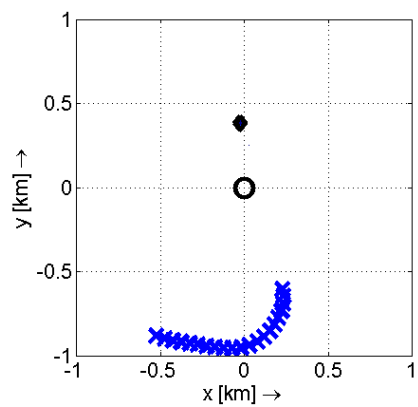

(c) iFilter, $k=22$
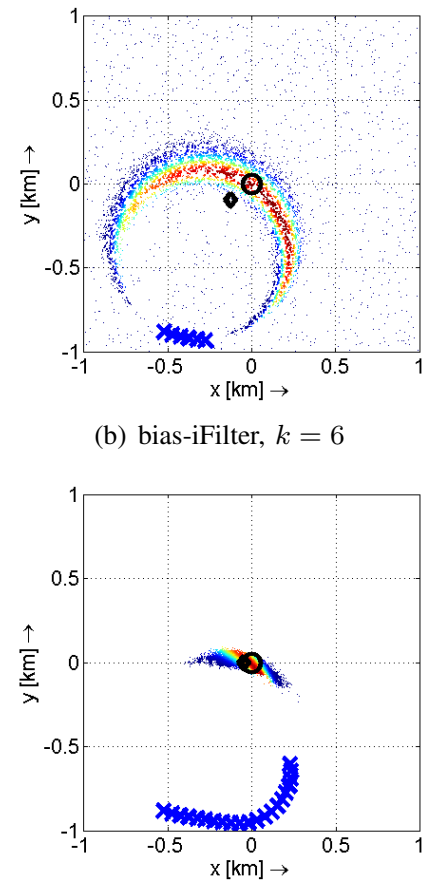

(d) bias-iFilter, $k=22$ (b) bias-iFilter, $k=6$

Fig. 3. Comparison of particle clouds; Sensor path (blue crosses), source location (black circle), particles and their weights (colored dots), and estimation result (black diamond)

${ }^{2}$ The required propabilities are initialized with $\psi_{0}(\mathbf{x} \mid \phi)=0.2, \psi_{0}(\phi \mid \phi)=$ $0.1, \psi_{0}(\phi \mid \mathbf{x})=0.01, p_{0}^{\mathrm{D}}(\mathbf{x})=0.9$, and $p_{0}^{\mathrm{D}}(\phi)=0.1$, and the intensity in $\phi$ with $f_{0 \mid 0}(\phi)=2$. The initialized and newly born particles are uniformly distributed in $\mathcal{S}=[-1 \mathrm{~km}, 1 \mathrm{~km}] \times[-1 \mathrm{~km}, 1 \mathrm{~km}] \times[-40 \mathrm{~m}, 40 \mathrm{~m}] \times$ $\left[-10^{\circ}, 10^{\circ}\right] \times\left[-15^{\circ}, 15^{\circ}\right]$. To predict the target intensity in Step 2, we use $\sigma_{\mathbf{p}}=2.5 \mathrm{~m}$ and $\sigma_{\mathbf{b}}=1^{\circ}$.

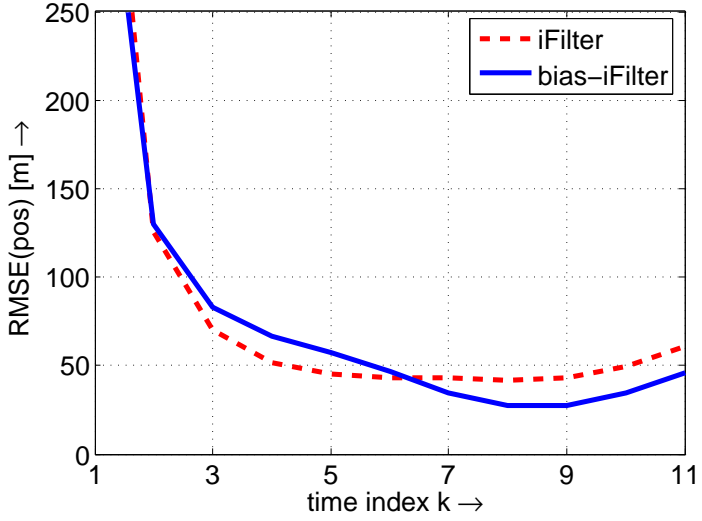

Fig. 4. Comparison of the RMSE of the localization results

In the following, all bearing measurements are used, but only the results of the first source are depicted, because the results of the other sources show a similar behavior. Fig. 4 compares the root mean square error (RMSE) of the localization results for the iFilter and the bias-iFilter. The results reveal that the bias compensation inherent in the biasiFilter approach significantly improves the localization results. The bias-iFilter needs longer to converge due to a higher dimensional state space.

\section{CONCLusions}

In this paper, we present a solution for the three-dimensional BOL problem for an unknown sensor bias and source number. An SMC version of the iFilter is used to solve the problem. The sensor bias can be compensated by using an augmented state vector containing the source location and the bias. As expected this leads to an improved localization performance. Finally, the proposed localization approach and results has been applied in flight experiments. We mention that the implemented algorithm is real-time capable.

\section{REFERENCES}

[1] K. Becker, "Target Motion Analysis (TMA)," in Advanced Signal Processing Handbook, S. Stergioulos, Ed., chapter 9, pp. 284-301. CRC Press, New York, NY, 2001.

[2] M. Gavish and E. Fogel, "Effect of bias on bearing-only target location," IEEE Trans. Aerosp. Electron. Syst., vol. 26, pp. 22-26, July 1990.

[3] Xiangdong Lin, Y. Bar-Shalom, and T. Kirubarajan, "Exact multisensor dynamic bias estimation with local tracks," IEEE Trans. Aerosp. Electron. Syst., vol. 40, pp. 576-590, Apr. 2004.

[4] M. Schikora, W. Koch, R. Streit, and D. Cremers, "Sequential Monte Carlo method for the iFilter," in 14th International Conference on Information Fusion, Chicago, USA, July 2011.

[5] M. Schikora, D. Bender, D. Cremers, and W. Koch, "Passive multiobject localization and tracking using bearing data," in 13th International Conference on Information Fusion, Edinburgh, UK, July 2010.

[6] R. L. Streit and L. D. Stone, "Bayes derivation of multitarget intensity filters," in 11th International Conference on Information Fusion, 2008.

[7] M. Oispuu and M. Schikora, "Multiple emitter localization using a realistic airborne sensor," in 14th International Conference on Information Fusion, Chicago, USA, July 2011.

[8] G. A. Carpenter and S. Grossberg, "ART 2: Self-organizing stable category recognition codes for analog input patterns," Applied Optics, vol. 26 , no. 23 , pp. 4919-4930, 1987.

[9] B. Ristic, D. Clark, and B.-N. Vo, "Improved smc implementation of the phd filter," in 13th International Conference on Information Fusion, Edinburgh, UK, July 2010. 